\title{
Producción chilena de molibdeno: influencia en el mercado mundial y su comportamiento exportador (2007-2016)*
}

\author{
Recibido: 30-01-2019 - Aprobado: 15-01-2020
}

Doi: https://doi.org/10.12804/revistas.urosario.edu.co/economia/a.8629

\author{
Ricardo Donoso Ovalle ${ }^{\dagger}$ \\ Bárbara Valenzuela Klagges $\ddagger$ \\ Andrés Soto Bubert ${ }^{\dagger}$ \\ Vlamir Muñoz Pavlov ${ }^{\dagger}$ \\ Iván Valenzuela Klagges ${ }^{\dagger}$
}

\section{Resumen}

Chile es líder en la industria mundial de molibdeno, sin embargo, no se observan estudios relacionados con la modelación del comportamiento exportador. Se estudia el comportamiento exportador chileno del molibdeno durante el período 2007-2016 y se detectan las variables que influyen en él. Adicionalmente, se identifica el efecto de las relaciones comerciales, tratados de libre comercio, facilitación comercial y logística. Para modelar el comportamiento exportador de molibdeno, se aplica un modelo gravitacional ampliado a la realidad del comercio bilateral chileno y al mercado mundial del molibdeno. El modelo planteado sigue un patrón que corresponde a datos de panel dinámicos.

Palabras clave: molibdeno, modelo gravitacional, Chile, exportaciones. Clasificación JEL: F13, F14.

* Investigación basada en la tesis de Ricardo Donoso Ovalle para optar al título profesional de Ingeniero Civil Industrial de la Facultad de Ingeniería y Tecnología de la Universidad San Sebastián.

$\dagger$ Facultad de Ingeniería y Tecnología, sede Bellavista, Universidad San Sebastián (Chile).

$\ddagger$ Unidad de Postgrado en Negocios, Universidad Mayor (Chile). Correo electrónico: barbara.valenzuela@umayor.cl

Cómo citar este artículo: Donoso, R., Valenzuela, B., Soto, A., Muñoz, V., \& Valenzuela, I. (2020). Producción chilena de molibdeno: influencia en el mercado mundial y su comportamiento exportador (2007-2016). Revista de Economía del Rosario, 23(1), 149-172. https:/ / doi.org/10.12804/revistas.urosario.edu.co/economia/a.8629 


\title{
Chilean Molybdenum Production: World-Market Influence and Its Exporter Behavio
}

\begin{abstract}
Chile is a leader in the global molybdenum industry; however, there are no studies related to the modeling of the exporting behavior. We studied the Chilean export behavior of molybdenum during the period 2007-2016 and detected the variables that influence it, in addition to identifying the effect of trade relations, free trade agreements, trade facilitation, and logistics. To model molybdenum's exporting behavior, an expanded gravitational model was applied to the reality of the Chilean bilateral trade and its world market. The raised model follows a pattern that corresponds to dynamic panel data.
\end{abstract}

Key words: Molybdenum, gravitational model, Chile, exports. JEL classification: F13, F14.

\section{Produção chilena de molibdênio: influência no mercado mundial e seu comportamento exportador (2007-2016)}

\section{Resumo}

O Chile é líder na indústria de molibdênio, no entanto, não se observam estudos relacionados com a modelação do comportamento exportador. Estuda-se o comportamento exportador chileno do molibdênio durante o período 2007-2016 e detectam as variáveis que influem nele. Adicionalmente, identifica-se o efeito das relações comerciais, tratados de livre comércio, facilitação comercial e logística. Para modelar o comportamento exportador de molibdênio aplica-se um modelo gravitacional ampliado à realidade do comercio bilateral chileno e mercado mundial do molibdênio. O modelo apresentado segue um patrão que corresponde a dados de painel dinâmico.

Palavras-chave: molibdênio, modelo gravitacional, Chile, exportações.

Classificação JEL: F13, F14. 


\section{Dinamismo del mercado mundial de molibdeno}

El molibdeno es un elemento metálico que no se encuentra en estado puro en la naturaleza, asociado generalmente a otras especies minerales como la molibdenita $\left(\mathrm{MoS}_{2}\right)$, powellita $\left(\mathrm{CaMoO}_{4}\right)$ y wulfenita $\left(\mathrm{PbMoO}_{4}\right)$. Sin embargo, solo la molibdenita posee las propiedades necesarias para el procesamiento mecánico y posterior obtención de molibdeno y sus derivados. El contenido de molibdeno en los cuerpos minerales viables varía entre el $0.01 \%$ y el $0.5 \%$ (Cochilco, 1992).

En Chile todo el molibdeno se obtiene como subproducto de la extracción de cobre. Se usa principalmente en la industria siderúrgica y, cuando se agrega al acero y a los hierros fundidos, el molibdeno mejora la resistencia, la templabilidad, la soldabilidad, la dureza, la resistencia a temperaturas elevadas y a la corrosión. En aleaciones a base de níquel, mejora la resistencia tanto a la corrosión como a la deformación por fluencia ${ }^{1}$ a altas temperaturas.

Las reservas mundiales de molibdeno estimadas en el año 2016 ascienden a 15 millones de TM, ${ }^{2}$ según la United States Geological Survey (USGS), lo que sugiere un aumento del $74 \%$ respecto al año 2007, donde solo se registraban 8,6 millones de TM. Los datos muestran que China (56\%), Estados Unidos ( $18 \%$ ) y Chile (12\%) concentran cerca del $86 \%$ de las reservas, lo que equivale a 12.9 millones de тм (Cochilco, 2017).

En cuanto a producción, en el año 2016 se alcanzó la cifra de 283.1 kTM, ${ }^{3}$ con China (128.9), Chile (55.6) y Estados Unidos (35.7) como los principales oferentes dentro del mercado mundial. Perú mantiene una importante cuarta posición con $25.8 \mathrm{kTM}$. El crecimiento de Chile y Perú en los últimos años (2013-2016) se debe principalmente al bajo cash cost o costo promedio de producción por libra de molibdeno, considerablemente menor que el de China y Estados Unidos, y siempre menor que el precio internacional del molibdeno (figura 1).

El molibdeno está cotizado en la Bolsa de Metales de Londres (LME, London Metals Exchange), alcanzando su precio máximo en la última década en el año 2007, con usD30.17/libra, cuando el precio de este metal era establecido por los productores, aunque registra una fuerte caída en el precio en 2009. En el año 2009 el molibdeno entró a la lista de metales cotizados en la LME, lo

1 Fluencia: deformación anormal que experimenta un material sometido a una carga o fuerza permanente y temperatura constante durante un período de tiempo. En los metales suele darse a temperaturas elevadas.

2 TM: toneladas métricas.

3 kTM: mil toneladas métricas. 
que explica una baja del 63\% respecto a 2007. A partir del año 2010 (USD15.8 / libra) se evidencia una tendencia a la baja en el precio internacional del molibdeno, salvo un pequeño repunte en 2014 (UsD11.39/libra), año donde se produjo una disminución en los inventarios de la LME. Cierra el año 2016 con usD6.48/libra (figura 2).

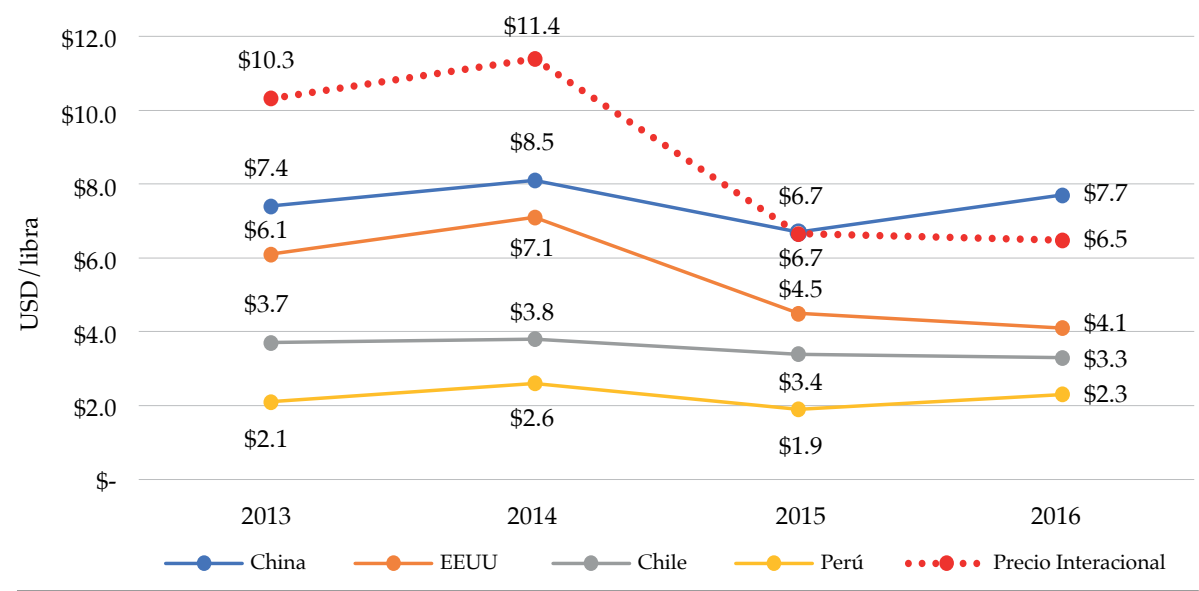

Figura 1. Cash cost promedio de producción por libra y precio internacional del molibdeno, 2013-2015

Fuentes: mercado internacional del molibdeno 2017 y Cochilco.

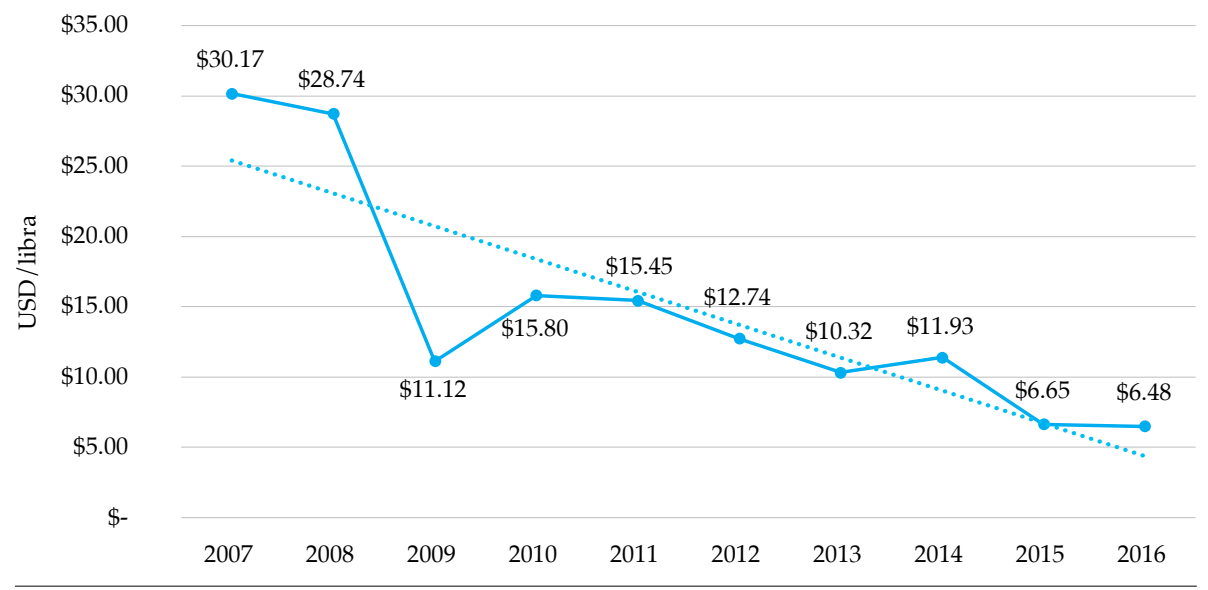

Figura 2. Precio del molibdeno usD/libra, período 2007-2016

Fuente: elaboración de los autores a partir de estadísticas de Cochilco.

El consumo mundial de molibdeno se registró en 287000 toneladas en 2016, un aumento del $2.5 \%$ respecto al período anterior. Durante 2007 y 2008, 
la Unión Europea se destacó como el mayor consumidor a nivel global, sin embargo, desde 2009 es desplazada a segundo lugar por China. Japón y Estados Unidos presentan un patrón de consumo similar (figura 3).

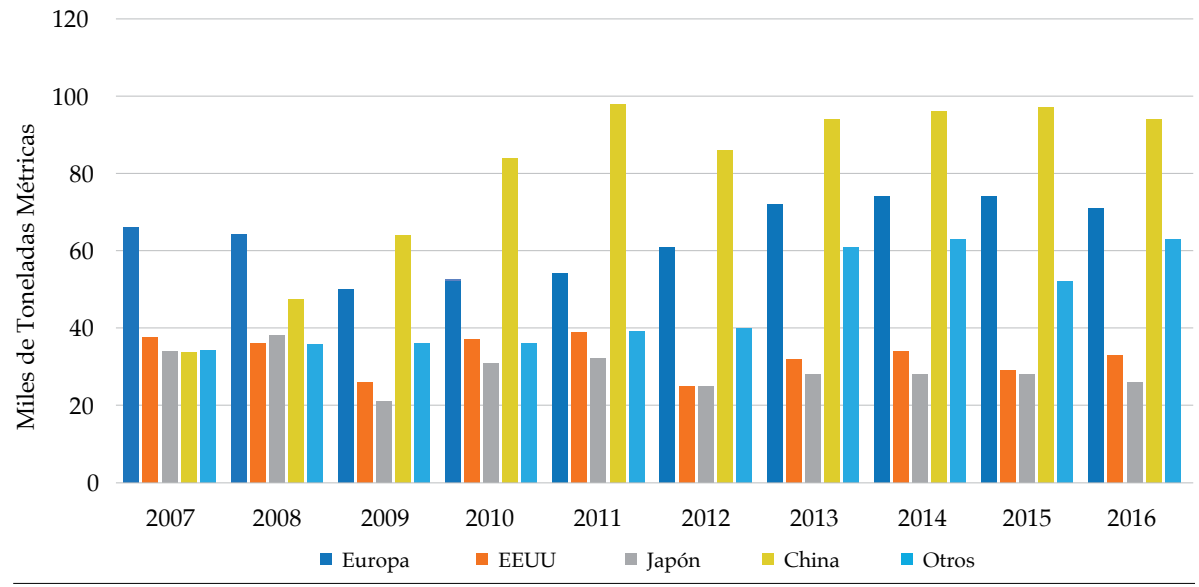

Figura 3. Consumo de molibdeno por región, período 2007-2016

Fuente: elaboración de los autores con base en estimaciones, proyecciones y datos de Cochilco.

Aproximadamente el $70 \%$ del molibdeno producido a nivel mundial se utiliza en la fabricación de aceros, entre los que se incluyen aleaciones (22\%), acero inoxidable $(19 \%)$, aceros de herramientas $(9 \%)$, aceros al carbono $(8 \%)$, hierro fundido ( $8 \%$ ) y superaleaciones (4\%) (figura 4 ). Estos aceros son usados en su mayoría en la industria mecánica, automotriz, construcción y petroquímica, por nombrar algunas.

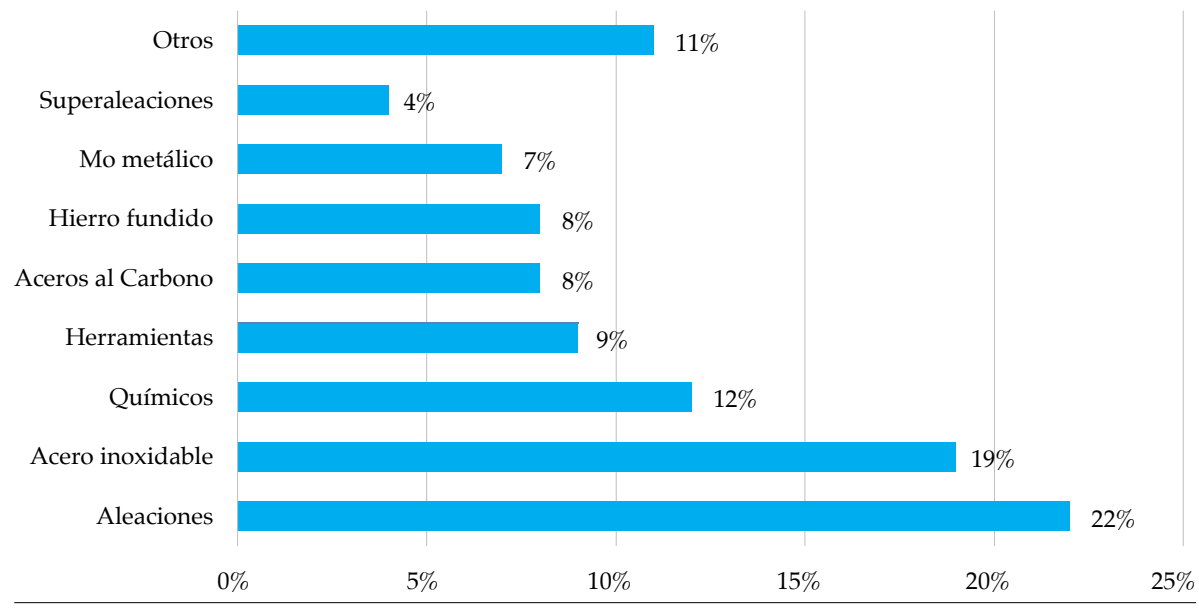

Figura 4. Participación en la demanda mundial por uso final, año 2016

Fuente: www.statista.com 
China es el mayor productor y consumidor de molibdeno a nivel global. El gigante asiático posee gran poder dentro del mercado mundial, es decir, cualquier pequeña variación respecto a su oferta o demanda tiene repercusión directa en el precio internacional y, por ende, también en la producción del resto de los países.

Chile, por su parte, aunque es un actor importante, posee un rol secundario dentro de la industria. Se ubica en tercer lugar en cuanto a reservas y ocupa el segundo puesto en producción, aportando el 19,6\% del total mundial, superado solo por China. Como se menciona anteriormente, en Chile todo el molibdeno se obtiene como actividad económica adicional a la extracción de cobre. Este tipo de instalaciones se encuentran desafectadas de las estimaciones del precio internacional del molibdeno, el cual se fija con base en proyecciones de producción realizadas sobre las plantas de producción primaria.

Actualmente, en Chile existen once instalaciones mineras que producen molibdeno en forma de concentrado. Cinco de estas operaciones son propiedad de Codelco, mientras que el resto son mineras privadas. Históricamente, la compañía que mayor aporte hace a la industria nacional es Codelco, que aportó el $55.1 \%$ de un total de $55647 \mathrm{TM}$ en el año 2016. Luego se presentan Sierra Gorda (18.6\%), Los Pelambres (12.9\%), Collahuasi (6.6\%), Los Bronces (4.5\%), Caserones (2.0\%) y Valle Central $(0.4 \%)$.

En 2018, son dos las empresas que se dedican a procesar y comercializar el molibdeno en Chile: Molymet y Molyb. Molymet, creada en 1975, es la más grande de las empresas procesadoras de molibdeno a nivel mundial, abarcando el $35 \%$ de la demanda mundial del metal (Molymet, 2018). Las instalaciones industriales de Molymet en Chile (Molymet Nos y Molynor) son capaces de procesar 66200 toneladas al año y ofrecen óxido de molibdeno técnico, ferromolibdeno y óxido de molibdeno puro. Por su parte, Molyb es una filial de Codelco que procesa los concentrados obtenidos en sus proyectos de extracción. La planta tiene una capacidad de producción anual de 15000 toneladas de óxido de molibdeno, de las cuales 4000 serán convertidas en ferromolibdeno.

Con el propósito de proyectar y disminuir la incertidumbre de la exportación chilena de molibdeno, surgen las preguntas de investigación que motivan el desarrollo de este estudio: ¿qué factores están influyendo en el comportamiento exportador de molibdeno en Chile?, ¿cuáles son sus proyecciones?

Dado todo lo expuesto y considerando la relevancia estratégica que tiene la minería en Chile, este estudio determina la influencia de la producción chilena de molibdeno en el mercado mundial y las variables que afectan su comportamiento exportador, durante el período 2007-2016. 
La motivación para realizar este análisis se presenta como consecuencia de la inexistencia de estudios que traten la modelación del comportamiento exportador chileno del molibdeno y sus proyecciones.

Para lograr el objetivo planteado, el trabajo se divide en tres secciones. La primera se centrará en el modelo y su metodología de estimación. La segunda sección expondrá los resultados de la estimación del modelo planteado. Para finalizar, conclusiones y recomendaciones.

\section{Estimación del modelo y metodología}

Contrario a lo que muchos economistas creen, la ecuación de gravedad tuvo su primera aparición en el siglo xIX con Ravenstein (1885) y muchos años más tarde con Zipf (1946), quienes la aplicaron a la migración y al movimiento de personas entre dos ciudades, respectivamente.

El primero en establecer una relación formal entre la ley de gravitación y el comercio es Tinbergen (1962) y luego Pöyhönen (1963), donde expresan el comercio bilateral $\left(T_{i, j}\right)$ entre países A y B como una función de sus masas (producto interno bruto o población) y su distancia geográfica, como proxy de los costos de transporte:

$$
T_{i, j} \alpha \frac{\left(P I B_{i}\right)^{\alpha}\left(P I B_{j}\right)^{\beta}}{\left(\text { Dist }_{i j}\right)^{\delta}}
$$

donde $\alpha, \beta, \delta \approx 1$. Los coeficientes $\alpha$ y $\beta$ representan el tamaño de las economías, mientras que $\delta$ representa el papel de la distancia geográfica. La ecuación 1 se puede representar de manera lineal empleando logaritmos naturales. La ecuación gravitacional aplicada para modelar el comportamiento exportador bilateral se sustenta en la ecuación 2:

$$
\ln \left(T_{i, j}\right)=\ln \left(P I B_{i}\right)+\ln \left(P I B_{j}\right)-\ln \left(\text { Dist }_{i j}\right)+\mu_{i}
$$

Donde:

$i, j:$ representan al país exportador e importador respectivamente.

$T_{i j}$ : flujo de exportación del país $i$ al $j$.

$P I B_{i, j}$ : producto interno bruto real del país $i$ y $j$ respectivamente.

Dist $_{i j}$ : distancia geográfica entre capitales de los países $i$ y $j$ en kilómetros. 
$\mu_{i}$ : error aleatorio, agrupa una serie de variables que influyen en el flujo comercial, pero no son observables.

Un poco más adelante, Anderson (1979), Bergstrand (1985) y Helpman (1985) obtienen ecuaciones de modelos de comercio internacional introduciendo los conceptos de diferenciación de los productos y en el rendimiento a escalas crecientes, brindando una potente base teórica. Mátyás (1997), Cheng y Wall (1999), Bayoumi y Eichengreen (1998) y Breuss y Egger (1999) introducen una serie de especificaciones econométricas al modelo gravitacional para evitar errores de estimación.

Variables explicativas como el PIB, tipo de cambio y variables ficticias o dummy como fronteras o idioma en común, existencia de tratados bilaterales o el uso de una misma moneda fueron introducidas por Frankel y Rose (2002) para analizar la relación entre los procesos de integración e incremento del comercio entre países.

Anderson y Van Wincoop (2003) plantean que la ecuación gravitacional tradicional no está bien especificada, ya que excluye términos de resistencia multilateral.

Santos Silva y Tenreyro (2006) obtienen una serie de conclusiones relacionadas con estimaciones obtenidas mediante la log-linealización de la ecuación de gravedad. En primer lugar, comprueban de manera empírica una de las realidades del comercio internacional: los países más pequeños tienden a ser más abiertos al comercio bilateral. En segundo lugar, plantean un problema relacionado con la analogía entre el comercio bilateral y la ley de gravitación de Newton, dado que en ocasiones la fuerza de atracción gravitacional es muy pequeña pero nunca toma el valor cero, en tanto que el intercambio comercial entre varios pares de países es inexistente y el valor cero es una realidad. Una de las soluciones para este problema es reemplazar las observaciones $T_{i, j}=0$ por $T_{i, j}=1$ o en su defecto reducir la muestra eliminando pares de países con cero-comercio, aunque ambas soluciones resultan insatisfactorias y pueden conducir a estimaciones incoherentes. En tercer lugar, establecen que todos aquellos modelos no linealizados mediante logaritmo natural poseen heterocedasticidad $\mathrm{d}^{4}$, por lo tanto, conducen a estimadores incoherentes.

4 Heterocedasticidad: se presenta cuando la varianza del error es distinta para cada valor de $x$. Provoca estimadores insesgados. Una forma eficiente de reducir la heterocedasticidad es aplicar la log-linealización de la ecuación, de esta manera los errores se distribuyen de forma normal sin importar el tamaño de la muestra. 
La denominada ecuación gravitacional se ha mantenido estable a lo largo del tiempo, con diferentes muestras de países y metodologías. Actualmente es uno de los modelos empíricos más estables y robustos de la economía.

En el último tiempo, el modelo gravitacional es bastante utilizado para analizar el comercio internacional, los flujos migratorios y la inversión extranjera, puesto que se adapta a la estimación mediante datos de panel estáticos y dinámicos. Teóricamente, a mayor tamaño de las economías del país exportador e importador mayor atracción gravitacional existirá entre ellos, mientras que a mayor distancia geográfica la atracción será menor. Tradicionalmente, los métodos estadísticos que intentan explicar un fenómeno observado a través de una serie de variables han sido tratados mediante regresiones lineales, usando el método de mínimos cuadrados ordinarios (MCO).

Labra y Torrecillas (2014) consideran que la metodología de estimación mediante datos de panel es una de las más usadas en los últimos años en el ámbito de la economía y los negocios, al permitir trabajar simultáneamente varios períodos de tiempo y los efectos individuales, $y$, a su vez, tratar el problema de la endogeneidad con datos de panel dinámicos. Para Cafiero (2005), la estimación del modelo gravitacional por medio de datos de panel mejora la cantidad de datos y calidad del análisis.

En este estudio, el modelo gravitacional linealizado (ecuación 2) es ampliado para adaptarse a la realidad de Chile, su comercio exterior y el mercado mundial de molibdeno representado en la ecuación 3:

$$
\begin{gathered}
T_{i j t}=\beta_{0}+\beta_{0}\left(Y_{i t}\right)+\beta_{2}\left(Y_{j t}\right)+\beta_{3}\left(d_{i j}\right)+\beta_{4}\left(\text { pmo }_{t}\right)+\beta_{5}\left(\text { prochimo }_{i t}\right) \\
+\beta_{6}\left(\text { prochicu }_{i t}\right)+\beta_{7}\left(\operatorname{cexp}_{i t}\right)+\beta_{8}\left(\operatorname{cimp}_{j t}\right)+\beta_{9}\left(\text { docimp }_{j t}\right) \\
+\beta_{10}\left(t c r_{i t}\right)+\beta_{11}\left(i d l_{i t}\right)+\beta_{12}\left(\text { infchn }_{t}\right)+\beta_{13}\left(\text { proac }_{t}\right) \\
+\beta_{14}\left(\text { exptecchn }_{t}\right)+\beta_{15}\left(\text { exptecjpn }_{t}\right)+\beta_{16}\left(\text { ppet }_{t}\right)+\sum_{h} \delta_{h} P_{i j}+\mu_{i j}
\end{gathered}
$$

Donde:

$i, j$ y $t$ : representan al país exportador, importador y año respectivamente.

$T_{i j t}$ : flujo de exportación del país $i$ al $j$ en el año $t$ en toneladas métricas.

$Y_{i t, j t}$ : producto interno bruto real (2010) del país $i$ y $j$ respectivamente en el año $t$ en USD.

$d g_{i j}$ : distancia geográfica entre capitales de los países $i$ y $j$ en kilómetros. 
$p m o_{t}$ : precio internacional del molibdeno en el año $t$ en USD/libra.

prochimo $_{i t}$ : producción chilena de molibdeno en el año $t$ en toneladas métricas.

prochicu $_{i t}$ : producción chilena de cobre en el año $t$ en toneladas métricas.

$\operatorname{cexp}_{i t}:$ costo de exportar por contenedor en el año $t$ en USD.

$\operatorname{cimp}_{i t}$ : costo de importar por contenedor en el año $t$ en USD.

$\operatorname{docimp}_{i t}:$ número de documentos necesarios para importar un contenedor.

$\operatorname{trc}_{i t}$ : tipo de cambio real $(1986=100)$ en Chile en el año $t$ en CLP/USD.

$i d l_{i t}$ : índice de desempeño logístico Chile ( 1 = bajo, 5 = alto).

infchn $n_{t}$ : inflación china en el año $t$ en porcentaje.

$\operatorname{proac}_{t}$ : producción mundial de acero en el año $t$ en toneladas.

exptecchn $n_{t}$ exportación de alta tecnología de China en el año $t$ en usD.

exptecjpn $n_{t}$ exportación de alta tecnología de Japón en el año $t$ en USD.

ppet $_{t}$ : precio del petróleo (Brent) en el año $t$ en USD/barril.

$\mu_{i}$ : error aleatorio.

Además, en la ecuación 2, se incluyen variables dummy o cualitativas indicadas como $\sum_{h} \delta_{h} P_{i j}$. Así, el estimador Mco entrega de forma separada el valor de los coeficientes de estos efectos. Estas variables asumen valor uno cuando se presenta el evento y cero cuando no se presenta. Las variables ficticias utilizadas son:

csp: crisis subprime del año 2008.

$i b$ : ingreso a la Bolsa de Metales de Londres en el año 2009.

$f c_{i j}$ : frontera común entre el país exportador y el país importador.

$i c_{i j}$ : idioma común entre el país exportador y el país importador. 
$c l$ - ue: intercambio comercial entre países miembros de la Unión Europea y Chile.

cl-corea: intercambio comercial entre Corea y Chile.

cl - usa: intercambio comercial entre Estados Unidos y Chile.

cl - chn: intercambio comercial entre China y Chile.

cl - jpn: intercambio comercial entre Japón y Chile.

cl-apec: intercambio comercial entre países miembros del Foro de Cooperación Económica Asia-Pacífico (APEC) y Chile.

cl - mercosur: intercambio comercial entre países miembros del Mercado Común del Sur (Argentina, Brasil, Paraguay, Uruguay y Venezuela) y Chile.

A partir del estudio elaborado en la primera etapa, surgen variables que tienen repercusión en el mercado mundial del molibdeno o en las exportaciones nacionales del metal. Entre estas variables se destaca el producto interno bruto (РІв) del país importador y exportador. En la figura 5 se observa la relación entre la producción mundial y las exportaciones físicas de molibdeno. Según lo esperado por el modelo gravitacional, el PIB, tanto del exportador como del importador, puede afectar positivamente las exportaciones de un determinado bien o servicio.

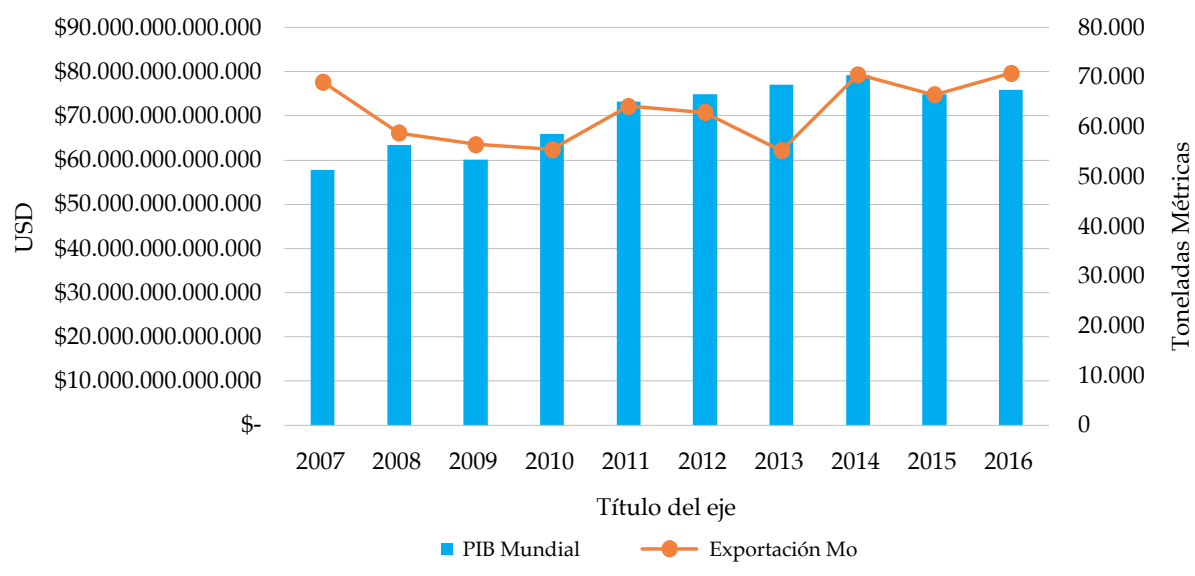

Figura 5. PIB mundial (USD) vs. exportación chilena de molibdeno (TM) Fuente: elaboración de los autores con base en datos del Banco Mundial y Cochilco. 
El precio internacional del molibdeno es fijado por la Bolsa de Metales de Londres (LME) con base en la oferta y demanda del mercado mundial, considerando únicamente a los productores primarios de molibdeno. Se incluye en la ecuación 2 para determinar su efecto en las exportaciones. Si bien un crecimiento en el precio produce una contracción de la demanda, es posible contrarrestar dicha disminución con un aumento en los ingresos percibidos por la exportación.

En Chile, todo el molibdeno que se produce se obtiene como subproducto o como una actividad económica adicional a la extracción de cobre, por lo que la producción nacional de molibdeno tiene directa relación con la producción de cobre.

A nivel mundial, tanto el costo de exportar como de importar siguen una tendencia al alza desde 2006. Por esto, se constituye como un importante y estratégico elemento de estudio, debido a las posibles perturbaciones o efectos que puede provocar en el curso de las relaciones comerciales.

La literatura señala que las exportaciones y el tipo de cambio real tienen una relación directa. Garcés (2006) expone que el tipo de cambio real tiene efecto positivo sobre la balanza comercial mexicana. Valenzuela Klagges (2015) en su estudio del comercio bilateral latinoamericano concluye que el tipo de cambio real tiene un efecto positivo y significativo en este mercado.

El índice de desempeño logístico es un indicador creado por el Banco Mundial con el objetivo de mostrar y describir las tendencias globales en materia de Logística. Se encarga de medir la eficiencia de las cadenas de suministro de cada país y como esta se desenvuelve en el comercio con otros países (socios comerciales). Una logística poco eficiente se refleja en un crecimiento de los costos del comercio y, por lo tanto, afecta la integración de los países en su afán de competir en el mercado global.

Como se menciona anteriormente, China es el mayor productor y consumidor de molibdeno. Petersen (2016) se basa en el estudio de Melitz (2003) para señalar que la tasa de inflación china puede afectar de forma indirecta a la estructura de exportaciones de un país, ya que, por lo general, una menor estabilidad macroeconómica tiende a aumentar la incertidumbre e induce una mayor concentración de exportaciones.

El $70 \%$ de todo el molibdeno que se produce a nivel mundial se utiliza en la industria siderúrgica para mejorar las propiedades mecánicas de los aceros, debido a esto, un aumento o disminución en la proyección de demanda de aceros tiene el mismo efecto en el mercado del molibdeno.

Se estima que el $17 \%$ del molibdeno producido a nivel mundial tiene un uso en la industria del petróleo y gas. La inversión en la industria petroquímica tiene directa relación con el precio internacional del crudo, por esto, 
se cree que cualquier variación en el precio del petróleo tendrá efecto en la demanda de molibdeno.

Las exportaciones de alta tecnología de un país están asociadas a productos que requieren un alto grado de investigación y desarrollo. Este indicador incluye productos que se encuentran dentro de categorías como equipos aeroespaciales, informáticos, instrumentos científicos y maquinaria electrónica.

Además, se incluyen en la ecuación 2 una serie de variables ficticias que permiten agregar y verificar la repercusión de ciertos eventos puntuales en las exportaciones de molibdeno y sus productos.

Siguiendo el estudio de Fuenzalida, Valenzuela y Corvalán (2018), se introduce en el modelo una variable relacionada con la crisis subprime, con el objetivo de analizar el efecto negativo de la crisis de 2008 en el precio del metal. Continuando la línea del trabajo mencionado, se incluyen una serie de acuerdos comerciales como: Tratado de Libre Comercio (TLC) entre Chile y China, Japón, Corea del Sur y Estados Unidos; Acuerdo de Complementación Económica (ACE) con el Mercosur; Acuerdo de Asociación Económica (ASE) entre la Unión Europea y Chile. Brito y Valenzuela (2015) señalan que el comercio intrabloque, por ejemplo, el Mercosur, tiene efecto positivo sobre el comercio bilateral de sus miembros.

Frontera e idioma común son variables que suelen introducirse en el modelo gravitacional para medir los efectos del transporte terrestre entre países limítrofes o la facilidad de comunicación por el idioma. Benavides, Olascoaga y Cuello (2015) señalan que el idioma común afecta de manera positiva las exportaciones del sector textil colombiano.

El modelo gravitacional empleado se calcula con base en las exportaciones físicas de molibdeno de tres productos principales: molibdenita, ferromolibdeno y óxido técnico. Se utiliza como pilar de cálculo la cantidad física, debido a la gran variabilidad del precio internacional del molibdeno en el período de estudio, detallado en la figura 2. Las bases de datos proporcionan la información de las exportaciones referente a los montos exportados y, para determinar las cantidades físicas, es necesario calcularlas con base en el precio internacional promedio anual, lo que da como resultado cierto grado de incertidumbre y una disminución en la cobertura de los datos reales (figura 6).

Para este estudio, se incluyen los 60 socios comerciales con los que Chile ha intercambiado productos de molibdeno (hasta 2016), que, según su zona geográfica, se distribuyen de la siguiente manera: 28 pertenecen a Europa; 16, a Asia; 10, a América; 4, a África; y 2, a Oceanía. Estos destinos representan en promedio el $88 \%$ del total de exportaciones físicas de molibdeno y sus productos durante el período 2007-2016, debido a lo expuesto anteriormente. Además, en esta investigación se cumple con la sugerencia de Roodman (2009), 
que propone usar una base de datos con un número de observaciones mayor a 100 y por un período de tiempo $(\mathrm{t})$ no mayor a quince años.

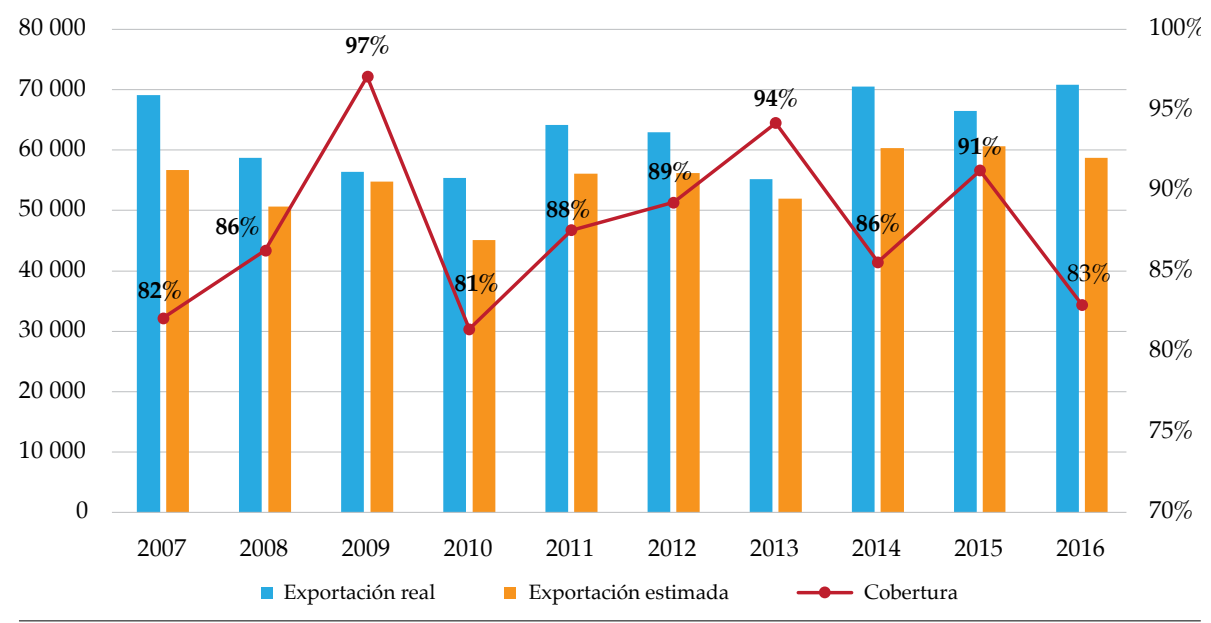

Figura 6. Exportación real vs. estimada

Fuente: elaboración de los autores con base en datos de la oEc y Cochilco.

Al usar un modelo gravitacional se toma una gran muestra de países y períodos, por lo que es posible que algunas observaciones asuman valor cero en un año determinado. Con el objetivo de abordar el problema de las observaciones cero en el comercio bilateral, Piani y Kume (2000), Zago de Azevedo (2001) y Wall (2003) han sustituido dichas observaciones por pequeños valores en la variable dependiente $(0,001)$. Algunos autores como Eichengreen e Irwin (1998) y Wall (2003) han experimentado con la sustitución de las observaciones cero por el valor 1, aunque esto último puede dar como resultado estimadores alterados o incongruentes.

Con el propósito de reducir la multicolinealidad se aplica el test de vif. El VIF es una medida de cuánto se incrementan las varianzas de los coeficientes estimados mediante Mco por la colinealidad entre las variables predictoras. En la práctica, el factor de inflación de la varianza es una medida de multicolinealidad potencial y de su intensidad en un análisis de regresión en Mco. Neter, Wasserman y Kutner (1990) indican que cuando el vif de un determinado coeficiente es mayor a 10 existen graves problemas de multicolinealidad. Una muestra cuyas variables presentan un vif menor o igual a 10 no está libre de multicolinealidad, pero entrega estimadores más precisos a medida que el vif tienda a 1 , su valor mínimo. Debido a que la multicolinealidad produce resultados inestables, las variables eliminadas por multicolinealidad se 
descartan para las siguientes estimaciones. A continuación, en la tabla 1 se muestran las variables descartadas por multicolinealidad.

Tabla 1. Variables descartadas por multicolinealidad según test de VIF

\begin{tabular}{llcc}
\hline \multicolumn{1}{c}{ Clave } & \multicolumn{1}{c}{ Variable } & VIF & vIF promedio \\
\hline proace & Producción mundial de acero & 868.51 & 642.95 \\
protecchn & Exportación de alta tecnología de China & 415.88 & 100.13 \\
cexp & Costo de exportar & 205.77 & 65.18 \\
tcr & Tipo de cambio real Chile & 186.25 & 57.43 \\
protecjpn & Exportación de alta tecnología de Japón & 44.44 & 14.71 \\
prochmo & Producción chilena de molibdeno & 10.55 & 4.60 \\
\hline
\end{tabular}

Fuente: elaboración de los autores.

Es importante destacar que una vez descartada la última variable prochmo, el vIF promedio $=2.76$ y todas las variables poseen un VIF $\leq 10$. Al descartar estas variables para siguientes estimaciones, la ecuación 3 se transforma en la ecuación 4:

$$
\begin{aligned}
T_{i j t}= & \beta_{0}+\beta_{1}\left(Y_{i t}\right)+\beta_{2}\left(Y_{j t}\right)+\beta_{3}\left(d_{g_{i j}}\right)+\beta_{4}\left(\text { pmo }_{t}\right)+\beta_{5}\left(\text { prochicu }_{i t}\right)+\beta_{6}\left(\operatorname{cimp}_{j t}\right) \\
& +\beta 7\left(\text { docimp }_{j t}\right)+\beta_{8}\left(i d l_{i t}\right)+\beta_{9}\left(\text { infchn }_{t}\right)+\beta_{10}\left(\text { ppet }_{t}\right)+\sum_{h} \delta_{h} P_{i j}+\mu_{i j}
\end{aligned}
$$

Otro de los aspectos problemáticos por considerar a la hora de evaluar un modelo es la heterocedasticidad. La heterocedasticidad se presenta cuando la varianza del error no es constante a lo largo de la muestra.

Al presentar heterocedasticidad los estimadores siguen siendo lineales, insesgados ${ }^{5}$ y consistentes, ${ }^{6}$ pero no son eficientes. ${ }^{7}$ En presencia de heterocedasticidad las varianzas del estimador no se pueden calcular.

La prueba más general para detectar la heterocedasticidad es el test de White. El test de White permite contrastar estimadores no lineales utilizando cuadrados y productos cruzados de todos los coeficientes. Este test funciona bajo la hipótesis $\left(H_{\mathrm{o}}\right)$ de la homocedasticidad y la hipótesis nula $\left(H_{a}\right)$ de la heterocedasticidad, en otras palabras, si el test de White arroja

5 Insesgado: el valor esperado del parámetro coincide con su valor real.

6 Consistente: una pequeña diferencia entre el valor real y el valor estimado por el modelo.

7 Eficiente: la desviación entre el valor real y el valor estimado será la menor posible. 
una Prob > chi $2=0.0000$ se cumple la hipótesis de la homocedasticidad y se descarta la hipótesis nula de heterocedasticidad.

Para esta estimación, el test de White calcula una Prob $>$ chi $2=0.0000$, por lo que se comprueba la hipótesis y se concluye que la muestra no presenta heterocedasticidad. A partir de esto se concluye que es posible aplicar la log-linealización de la ecuación para reducir los residuos de la estimación. La log-linealización de la ecuación permite distribuir los errores de manera normal sin importar el tamaño de la muestra.

Una vez verificado que la utilización de logaritmos naturales en la ecuación reduce el residuo, se procede a log-linealizar la ecuación 5:

$$
\begin{gathered}
\ln \left(T_{i j t}\right)=\beta_{0}+\beta_{1} \ln \left(Y_{i t}\right)+\beta_{2} \ln \left(Y_{j t}\right)+\beta_{3} \ln \left(d g_{i j}\right)+\beta_{4} \ln \left(p m o_{t}\right) \\
+\beta_{5} \ln \left(\text { prochicu }_{i t}\right)+\beta_{6} \ln \left(\text { cimp }_{j t}\right)+\beta_{7} \ln \left(\text { docimp }_{j t}\right) \\
+\beta_{8} \ln \left(\text { idl }_{i t}\right)+\beta_{9} \ln \left(\text { infchn }_{t}\right)+\beta_{10} \ln \left(\text { ppet }_{t}\right)+\sum_{h} \delta_{h} P_{i j}+\mu_{i j}
\end{gathered}
$$

Como una forma de corregir la endogeneidad e incorporar relaciones de causalidad que se generan en el modelo, se recomienda utilizar datos de panel dinámicos. Una de las maneras más frecuentes para reducir la endogeneidad ${ }^{8}$ es a través de retardos de la variable dependiente. Siguiendo el estudio de Fuenzalida et al. (2018), el cual comprueba el dinamismo del comportamiento exportador chileno de bienes y servicios, se sugiere utilizar datos de panel dinámicos para modelar las exportaciones de molibdeno.

La ecuación 5 se adapta para realizar la estimación con datos de panel dinámicos representada por la ecuación 6:

$$
\begin{gathered}
\ln \left(T_{i j t}\right)=\beta_{0}+\beta_{1} \ln \left(T_{i j t-1}\right)+\beta_{2} \ln \left(Y_{i t}\right)+\beta_{3} \ln \left(Y_{j t}\right)+\beta_{4} \ln \left(\text { pmo }_{t}\right) \\
+\beta_{5} \ln \left(\text { prochicu }_{i t}\right)+\beta_{6} \ln \left(\text { cimp }_{j t}\right)+\beta_{7} \ln \left(\text { docimp }_{j t}\right) \\
+\beta_{8} \ln \left(\text { idl }_{i t}\right)+\beta_{9} \ln \left(\text { infchn }_{t}\right)+\beta_{10} \ln \left(\text { ppet }_{t}\right)+\sum_{h} \delta_{h} P_{i j}+\mu_{i j}
\end{gathered}
$$

Donde $T_{i j t-1}$ es el primer retardo del flujo de exportación del país $i$ al país $j$ de la variable dependiente. En relación con la metodología mediante datos de panel dinámico, Perroti (2015) plantea que en la estimación del modelo gravitacional dinámico aplicado a los flujos de comercio debe recurrirse al uso

8 Endogeneidad: es un lazo de causalidad entre las variables independientes $(x) y$ las variables dependientes (y) de un modelo. Puede surgir debido a errores en la medición o a variables omitidas (e) que poseen relación con las variables independientes de modo que $\operatorname{cov}(x, e) \neq 0$ 
de Arellano y Bond (1991), dado que los estimadores de mínimos cuadrados ordinarios (MCO), efectos fijos y efectos aleatorios resultan sesgados e inconsistentes. Al estimar mediante la fórmula de Arellano y Bond (1991), en el que se utilizan los rezagos de la variable dependiente como instrumentos para su estimación, se emplea el método generalizado de momentos para la estimación de un modelo dinámico, lo que permite obtener resultados consistentes. Para Labra y Torrecillas (2014), la metodología de datos de panel es una de las más usadas en economía y negocios en los últimos tiempos, puesto que su fortaleza es que permite trabajar simultáneamente varios períodos de tiempo y efectos individuales, $y$, a su vez, permite tratar el problema de la endogeneidad y asumir los efectos de variables omitidas.

En este estudio, se siguió la metodología planteada por Perroti (2015) al descartar aquellas variables que no evidenciaban la significatividad estadística a niveles usuales. Por lo tanto, en los resultados que se presentan a continuación solo se exponen aquellas variables con buen comportamiento estadístico.

Este resultado solo explica el comportamiento exportador chileno en el molibdeno. Variados estudios han aplicado el modelo gravitacional para modelar el comportamiento exportador y/o importador de un único producto o varios productos sin presentar errores de estimación o limitaciones (Jacobo, 2010; Fuenzalida O'Shee \& Valenzuela Klagges, 2019).

\section{Resultados}

En la tabla 2 se detallan los resultados obtenidos luego de realizar la estimación con datos de panel dinámicos con un retardo de la variable dependiente bajo la técnica de Arellano-Blundell.

Los resultados indican que el retardo de la variable dependiente es significativo y positivo.

Se concluye que las exportaciones de molibdeno de un determinado período tienen efecto positivo y significativo en las exportaciones del período siguiente.

Se evidencia que el coeficiente del pIB del país exportador, en este caso Chile, es positivo y altamente significativo ( $0 \%$ error) siguiendo la teoría del modelo gravitacional. Sin embargo, el PIB del país importador no es relevante para las exportaciones chilenas de molibdeno, contrario a la teoría del modelo gravitacional pero consistente con las características económicas de los commodities expuestas en la sección 2. 
Tabla 2. Resultados de la estimación de la ecuación 4

\begin{tabular}{cc}
\hline Variable & Efecto dinámico: Arellano-Bover/Blundell-Bond (7) \\
\hline Ln (exp -1) & $0, .2080515(0.0574603)^{* * *}$ \\
Ln (PIB exportador) & $9.934768(2.746201)^{* * *}$ \\
Ln (distancia geográfica) & $55.15939(16.86534)^{* *}$ \\
Ln (prod. chilena cobre) & $-16.26692(5.791336)^{* *}$ \\
Inicio-término & $-2.407559(0.5215666)^{* * *}$ \\
Frontera común & $-273.2167(67.77133)^{* * *}$ \\
Idioma común & $86.7541(26.88601)^{* *}$ \\
Chile-Unión Europea & $41.71928(12.96635)^{* *}$ \\
Chile-China & $157.0155(56.3946)^{* *}$ \\
Chile-Corea del Sur & $618.2698(112.4057)^{* * *}$ \\
Constante & $-546.915(208.759)^{* *}$ \\
Número de observaciones & 540 \\
\hline
\end{tabular}

Nota 1: valores con heterocedasticidad corregida.

Nota 2: nivel de significancia: ${ }^{* *}=0 \%$ error; ${ }^{* *}=0 \%<\mathrm{P} \leq 2.5 \%{ }^{*}=2.5 \%<\mathrm{P} \leq 5 \%$.

Nota 3: los valores entre paréntesis representan el estándar de error.

Fuente: elaboración de los autores.

Por otra parte, la distancia geográfica posee coeficiente positivo, también contraria a la teoría del modelo gravitacional, aunque consistente con las características de los commodities. Adicionalmente, gran parte de las exportaciones de molibdeno tienen como destino países asiáticos y europeos, esto explica el coeficiente de la distancia geográfica y el efecto negativo y significativo de la frontera común.

El coeficiente negativo de la producción chilena de cobre (contrario a lo que indica la realidad) se debe principalmente a la omisión de variables que tienen relación o son explicativas de la producción nacional de cobre y del comportamiento de su mercado, que el software asume como relaciones de causalidad al momento de estimar los coeficientes, pero que no afectan al modelo como tal.

Aunque dentro de la muestra solo 8 de los 60 socios comerciales son de habla hispana, el poseer un idioma común incrementa de manera importante las exportaciones chilenas de molibdeno.

Los acuerdos comerciales existentes entre Chile y la Unión Europea, y China y Corea del Sur son positivos y significativos. Estos resultados muestran la importancia de los acuerdos con miembros de la Unión Europea, así como también los tratados de libre comercio (TLC) firmados con los grandes 
protagonistas del continente asiático. A pesar de ser el principal destino de las exportaciones de molibdeno, el TLC entre Chile y Japón no resulta significativo en el modelo estimado. Una razón posible es que el tratado se firma justamente en el año 2007, por lo que aún no es posible percibir de manera empírica el efecto del acuerdo en el comercio bilateral y, específicamente, en las exportaciones de molibdeno. Otros acuerdos de complementación económica como el Mercosur o APEC no presentan significancia dentro del período de estudio.

El precio del molibdeno, el costo de importar y los documentos necesarios para importar no resultan significativos dentro del modelo, ya que se trata de un commoditie. Los commodities mineros son bienes estratégicos que por ser volátiles pueden generar altos retornos, debido a esto los importadores están dispuestos a asumir los costos de transporte con el fin de obtener mayores ganancias y otorgar mayor valor agregado. Por esta razón, el índice de desempeño logístico no posee un rol importante dentro del proceso de exportación.

Tampoco resultan significativos los coeficientes correspondientes al precio del petróleo y la inflación de China. El índice de inflación china no resulta significativo para las exportaciones porque el nivel de precios está muy controlado y no supera el $4 \%$. El precio del petróleo no tiene efecto directo en la producción chilena de molibdeno, pero sí en la producción nacional de cobre, dado que el precio del petróleo y del cobre tienen una alta correlación, sin embargo, sus consecuencias son asumidas por el retardo de la variable dependiente.

El coeficiente de la crisis subprime no resulta significativo. Esta variable intenta medir el efecto de la crisis en el año 2009, pero no considera el efecto del año 2008 como inicio donde ya se presentaban contracciones de consumo e inversión en Estados Unidos y desaceleración económica a nivel mundial.

El ingreso a la Bolsa de Metales de Londres (LME) en el año 2009 no afecta las exportaciones de molibdeno. Esto se debe básicamente a que el precio que fija la LME está calculado según las proyecciones de producción de los productores primarios, mientras que Chile es un productor $100 \%$ secundario, es decir, la producción de molibdeno depende netamente de la extracción de cobre y no de otros factores de mercado. Esto último también explica la falta de significancia de la variable que representa el precio del petróleo; si bien un alza en el precio del petróleo provoca un aumento de la inversión en la industria del petróleo y gas, es un factor de mercado externo del cual Chile se encuentra exento.

A pesar de las limitaciones planteadas en la sección 1, se logra obtener un modelo que sigue una tendencia muy similar a la real. En la figura 7, se muestra la comparación entre las exportaciones reales de molibdeno y sus 
productos y los resultados obtenidos mediante la estimación de datos de panel dinámicos con un retardo.

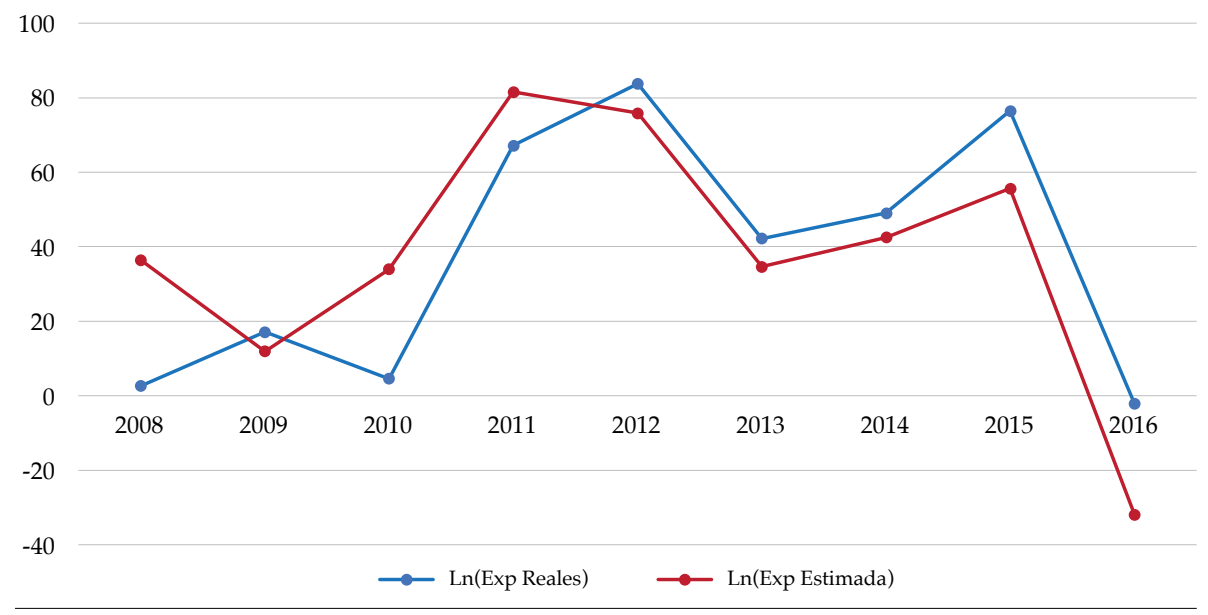

Figura 7. Exportaciones reales vs. exportaciones estimadas

Fuente: elaboración de los autores con base en resultados del modelo y estadísticas de Cochilco.

La figura 7 refleja el período 2008-2016 para tomar en cuenta el efecto de las exportaciones del año 2007 en adelante. La importante diferencia observada en los años 2008 y 2010 entre lo real y lo estimado se explica por:

- 2008: ausencia de la variable de comienzo de la crisis subprime, año 2008. La crisis subprime fue una crisis financiera que afectó el sector inmobiliario de Estados Unidos; este tipo de crisis son altamente contagiosas y adquieren nivel mundial en poco tiempo.

- 2010: ausencia de la variable relacionada con el precio internacional del cobre, el cual alcanza uno de sus puntos más altos (UsD0.42/libra), luego de sufrir la segunda peor caída en el precio en la década de estudio en el año 2009 (USD0.23/libra). Este aumento en el precio del cobre provoca un alza significativa en la oferta del metal y un incremento en el nivel de producción de molibdeno como subproducto. La volatilidad del precio del cobre influye en la producción nacional de cobre y posterior producción de molibdeno.

\section{Conclusiones y recomendaciones}

A partir del estudio de mercado se evidencia que Chile es líder en el mercado mundial de molibdeno, tanto en producción como en reservas, sin embargo, 
en su condición de productor secundario no tiene influencia sobre el precio internacional. Para Gomero (2019), los commodities son productos transables cuya exploración y explotación y posteriores exportaciones son atribuibles a empresas mineras, cuyo capital en su mayoría en Suramérica son extranjeros, estando los precios anclados a los vaivenes del mercado internacional, específicamente de las acciones que realizan las dos economías más importantes del mundo, como son Estados Unidos y China. En este sentido y según lo expuesto por Socha y Mejía (2016), la política fiscal chilena es considera contracíclica, pues aísla el efecto cíclico de tres variables para estimar el ingreso y el gasto público coherentes con los ingresos estructurales. Estas tres variables son la actividad económica, el precio del cobre y el precio del molibdeno. La estimación del precio de referencia del molibdeno es calculada sobre la base de criterios históricos (Rodríguez, Tokman \& Vega, 2006).

El mercado del molibdeno en su conjunto está estrechamente vinculado a China y Chile no es la excepción, así lo refleja el coeficiente positivo y significativo del TLC Chile-China. El mercado mundial depende en gran parte de China, no solo porque el país produce gran parte del metal. El gigante asiático posee un sector industrial masivo que requiere enormes cantidades de molibdeno para fabricar acero y productos tecnológicos. A pesar del cierre de algunas operaciones luego de las fuertes inspecciones efectuadas por el gobierno en el contexto del cuidado ambiental, el gigante asiático no ha visto disminuida su producción, la cual se mantiene prácticamente constante durante el período 2015-2016. Se sugiere en futuras investigaciones profundizar el modelo de fomento impulsado por el gobierno chino y evaluar la factibilidad de replicarlo en el contexto chileno.

La producción nacional de molibdeno es muy poco diversificada y totalmente dependiente de la producción de cobre y de sus factores de mercado. Solo existen tres plantas en suelo nacional dedicadas a la maquila y procesamiento de molibdeno, por lo que las oportunidades de investigación y desarrollo de nuevos productos que satisfagan otros segmentos de mercado son limitadas.

Durante el período 2016 las exportaciones de molibdeno y sus productos ascendieron a la cifra de USD921 millones. Los principales destinos de las exportaciones se encuentran en Asia (46\%) y Europa (31\%), siendo los países más importantes Japón (23\%), Corea del Sur (17\%) y Estados Unidos (15\%). Dentro de los productos de molibdeno, el más vendido es el dióxido de molibdeno, seguido por el concentrado de molibdenita, trióxido de molibdeno y ferromolibdeno. El dióxido de molibdeno y el concentrado de molibdenita se exportan generalmente para ser procesados por los países de destino y obtener otros productos de interés, o bien utilizarlos de forma directa. Por 
otro lado, el trióxido de molibdeno y el ferromolibdeno son empleados en la producción de aceros.

Las exportaciones mineras representan más del $50 \%$ del total de exportaciones chilenas durante todo el período de estudio, mostrando una alta dependencia del cobre y sus derivados. Chile se caracteriza por ser un país exportador de materias primas, esto representa una debilidad latente en el comercio bilateral chileno y, por consiguiente, de la economía en general. Esta realidad debe ser modificada en el corto-mediano plazo, fomentando la manufactura de bienes más desarrollados y la innovación en la industria nacional, diversificando el portafolio de productos, otorgando valor agregado a las materias primas e incentivando la inversión en tecnología.

Los resultados obtenidos permiten concluir que el comportamiento exportador chileno de molibdeno es dinámico y oscilante, siendo este resultado coincidente con el de Fuenzalida et al. (2018). Esto implica que la cantidad exportada en un determinado período anual tiene un efecto incremental correspondiente al $20.8 \%$ de las exportaciones del año siguiente.

El coeficiente de la producción chilena de cobre es negativo, contrario a lo que refleja la realidad. La negatividad del estimador es producto de relaciones de causalidad encontradas por el software luego de omitir variables relacionadas con el mercado del cobre, como, por ejemplo, el precio. Al ser un commoditie, al igual que el molibdeno, el cobre posee las mismas características de mercado, aunque su efecto en las exportaciones chilenas es mucho más importante. Debido a que es necesario para la producción de molibdeno, se recomienda en futuras investigaciones incluir variables que rigen el mercado mundial de cobre y verificar su efecto en las exportaciones de molibdeno.

Se confirma que mientras mayor sea el producto interno bruto del país importador, mayor será la exportación de molibdeno. Además, los resultados muestran que la relación entre distancia geográfica y exportación de molibdeno es directa, esto se explica debido a que gran parte de los socios comerciales de Chile en materia de exportaciones de molibdeno se encuentran en Asia y Europa, razón por la cual el coeficiente correspondiente a la frontera en común es negativo.

Asociado con lo anterior, las relaciones comerciales entre Chile y miembros de la Unión Europea, los TLc Chile-China y Chile-Corea del Sur, tienen coeficientes altos y positivos, sobre todo este último. En su defecto, se descartan como significativos los siguientes acuerdos bilaterales: Chile-Estados Unidos, Chile-Japón, Chile-APEC y Chile-Mercosur.

En cuanto a las hipótesis planteadas en la sección 1, solo resulta significativo el producto interno bruto (PIB) del país exportador y los tratados de libre comercio (TLC) entre Chile-Corea del Sur, Chile-China, y acuerdos comerciales 
entre Chile y los miembros de la Unión Europea; todos ellos positivos. La producción chilena de cobre afecta negativamente a la exportación chilena de molibdeno. Por otro lado, la distancia geográfica influye de forma positiva al nivel de exportaciones, por las razones mencionadas en párrafos anteriores. El costo de exportar no resulta significativo dentro de la estimación. Las hipótesis planteadas basadas en la teoría del modelo gravitacional de comercio y en la literatura consultada se cumplen de manera parcial con ciertas excepciones.

\section{Referencias}

Anderson, J. (1979). A theoretical foundation for the gravity equation. American Economic Review, 69(1), 106-116.

Arellano, M., \& Bond, S. (1991). Some tests of specification for panel data: Monte Carlo evidence and an application to employment equations. The Review of Economic Studies, 58(2), 277-297.

Bergstrand, J. (1985). The gravity equation in international trade: some microeconomic foundations and empirical evidence. The Review of Economics and Statistics, 71, 143-153.

Cochilco. (1992). El molibdeno.

Cochilco. (2017). Anuario de estadísticas del cobre y otros metales 1997-2016.

Frankel, J., \& Rose, A. (2002). An estimate of the effect of common currencies on trade and income. The Quarterly Journal of Economics, 117(2), 437-466.

Fuenzalida, D., Valenzuela, B., \& Corvalán, A. (2018). La facilitación comercial y sus efectos en el comercio bilateral chileno de 2006 a 2014. Revista Cepal, (124), 173-191.

Fuenzalida O'Shee, D., \& Valenzuela Klagges, B. (2019). Extractivismos versus exportaciones de manufacturas en Sudamérica: un desafío pendiente. El Trimestre Económico, 86.341, 127-144.

Garcés, D. (2006). La relación de largo plazo del PIв mexicano y sus componentes con la actividad económica en Estados Unidos y el tipo de cambio real. Economía Mexicana. Nueva Época, XV(1), 5-30.

Jacobo, A. D. (2010). Una estimación de una ecuación gravitacional para los flujos bilaterales de manufacturas Mercosur-Unión Europea. Economía Aplicada, 14.1, 67-79.

Labra, R., \& Torrecillas, C. (2014). Guía cero para datos de panel. Un enfoque práctico. Working Paper 16. Madrid: Universidad Autónoma de Madrid.

Molymet. (2018). Press realease 2018: resultados del segundo trimestre.

Neter, J., Wasserman, W., \& Kutner, M. H. (1990). Regression, analysis of variance, and experimental design. Applied Statistical Models, 614-619. 
Rodríguez, J., Tokman, C., \& Vega, A. (2006). Política de balance estructural: resultados y desafíos tras seis años de aplicación en Chile. Santiago de Chile: Estudios de Finanzas Públicas, Dirección de Presupuestos, Gobierno de Chile.

Pöyhönen, P. (1963). A tentative model for the volume of trade between Countries. Weltwirtschaftliches Archiv, 90, 93-100.

Ravenstein, E. (1885). The laws of migration. Journal of the Statistical Society of London, 48(2), 167-235.

Roodman, D. (2009). A note on the theme of too many instruments. Oxford Bulletin of Economics and Statistics, 71(1), 135-158.

Silva, J. S., \& Tenreyro, S. (2006). The log of gravity. The Review of Economics and Statistics, 88(4), 641-658.

Socha, D. G., \& Mejía, M. H. (2016). Buenas prácticas de ahorro e inversión pública en Chile y Noruega, producto de la renta de recursos naturales: fondos soberanos de inversión. Divergencia, (21), 41-57.

Tinbergen, J., \& Hekscher, A. (1962). Shaping the world economy. Suggestions for an-international economic policy. Twentieth Century Fund.

United States Geological Survey (usgs). (2008-2017). Mineral commodity summaries.

Zipf, G. K. (1946). The $\mathrm{P}_{1} \mathrm{P}_{2} / \mathrm{D}$ hypothesis: on the intercity movement of persons. American Sociological Review, 11, 677-686. 